\title{
Quad-spectral Unaided Experimental Scanner of Topography on BEXUS 27
}

\author{
Ric Dengel, Nico Florschütz, Valentin Huber, Tim \\ Muller, Jan von Pichowski, Alexander Rabinowitsch, \\ Sebastian Scholz, Hans Schülein, Eike Steinweg, \\ Benjamin Stippel, Peter Stöferle, Isabell Wittekind, \\ Oliver Wizemann, Alexander Zaft, and Lukas Zembrot \\ Institut für Informatik - Lehrstuhl VIII \\ Julius-Maximilian University Würzburg \\ Würzburg, Germany \\ ric.dengel@outlook.com
}

\author{
Dominik Woiwode \\ Gottfried Wilhelm Leibniz University Hanover \\ Hanover, Germany \\ Katrin Griebenow \\ Johannes Gutenberg University Mainz \\ Mainz, Germany
}

\begin{abstract}
This paper describes the REXUS/BEXUS experiment for Quad-spectral Unaided Experimental Scanner of Topography (QUEST). QUEST as part of the REXUS/BEXUS program was designed, developed, built, tested and operated by a team of 17 students from different German universities. It scanned the planet surface by analysing an array of four light sensors (RGB and IR) and two spectrometers. A reusable cluster algorithm determined autonomously onboard an overview image of the surface with marked areas depending on the type of the surface. Furthermore, the algorithm's data base was generated and optimized before and during flight. Regarding the hardware a modular sensor framework with standardized interfaces was implemented. The project has been a successful step to the designated target to build an autonomous system which could be used in interplanetary missions with demanding constraints on the bandwidth.
\end{abstract}

Keywords-Autonomous;

Topography

Scanner; REXUS/BEXUS

\section{INTRODUCTION}

A key feature of interplanetary satellite missions is the analysis of the planet surface. Therefor many different sensors are used to get a detailed overview. Nevertheless, the bandwidth for transferring the measurements is limited in a satellite mission. Thus, autonomous evaluation and election of valuable information on board of the satellite is required to reduce the resulting data size. There are several kinds of observations made by satellites from the surface of a planet. An interesting research area is the categorization of distinct areas on the surface. However, new algorithms to perform this kind of research are rare. The overall purpose of this experiment is to contribute to landscape categorization based on satellite imaging with a focus on the requirements of interplanetary missions. These requirements are reflected in a modular system made for reusability and result in a testbed for a newly developed algorithm for landscape distinction based on cluster analysis of distinct sensor and camera measurements. The Quad-spectral Unaided Experimental Scanner of Topography (QUEST) mission is based on the following primary scientific objectives:

- Development of a modular sensor framework

- Taking pictures of the earth in different wavelengths

- Differentiation of landscapes based on taken images

- Onboard data analysis

\section{EXPERIMENT DESCRIPTION}

\section{A. Experiment Concept}

To properly categorize the landscape the algorithm needs a set of predefined data. Therefore, it is necessary to obtain reference data with the given sensors and cameras to calibrate the algorithm. This raw data was collected in previous experiments on the ground, the ascent phase of the balloon and while the final experiment on the balloon is running. After the raw data is collected some areas are categorized by hand. Therefor an easy to use calibration software is produced and integrated in the ground station. This algorithm does not restrict the number and kinds of sensors and cameras. An ordinary RGB camera, an IR camera and a spectrometer are used. Those sensors are giving us the possibility to distinguish between snow, vegetation, water, rocks and overlaying clouds. To keep the possibility for late sensor switches an open modular sensor framework is designed as shown in Fig. 1.

Every sensor is coupled to a pre-processing unit to provide clearly defined data on a common data bus for all sensors. The data is requested by the main processor and fed to the algorithm. The result is stored and periodically send to the ground station. Additionally, data logging is performed and stored for post experiment analysis of the system behaviour. 


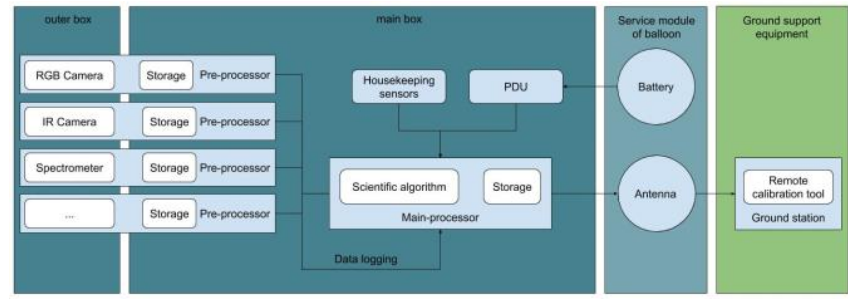

Fig. 1. Experiment Concept

\section{B. Machnics Design}

The experiment is composed of two main units; on the first hand the sensor box, which is located outside of the gondola containing all the sensors, cameras and the corresponding preprocessors. On the other hand, the gondola box, which contains the power distribution, the main processor and serves as the link between the gondola and the experiment. The shifted sensor box is necessary to provide an unobstructed field of view downwards. The whole box has a dimension of $821 \times 280$ $\mathrm{x} 380 \mathrm{~mm}$ and a mass of $8.5 \mathrm{~kg}$. The experiment is not significantly optimized in aspect of volume or mass.

Since the thermal environment on a high-altitude balloon mission is very harsh a proper thermal control was implemented. For this purpose, several heat sinks were mounted onto the processors and power converter to prevent the system from overheating. Furthermore, thermal insulation in form of high-density polyethylene was integrated. Also, an active thermal control system was integrated to prevent the spectrometer from freezing, which is the most thermal sensitive part.

\section{Electronics Design}

The experiment unit consists of nine components, separated in inner and outer components. On the outside there is the sensor box including two near infrared cameras, two visual light cameras and two mini spectrometers. The cameras are paired with a pre-processing unit including a GUMSTIX IceStorm board. For the cameras a redundant design is used to collect more data and increase reliability.

Inside the gondola there are the other electronic components: pre-processing boards for the spectrometers, the Onboard Computer (OBC) and the Power Distribution Unit (PDU). The PDU provides for the whole system which runs on two different voltage levels. It converts the provided battery power to the necessary $3.3 \mathrm{~V}$ and $5 \mathrm{~V}$. As mentioned in the mechanical section a thermal control system is attached to the spectrometer. Also, a housekeeping system is integrated to get

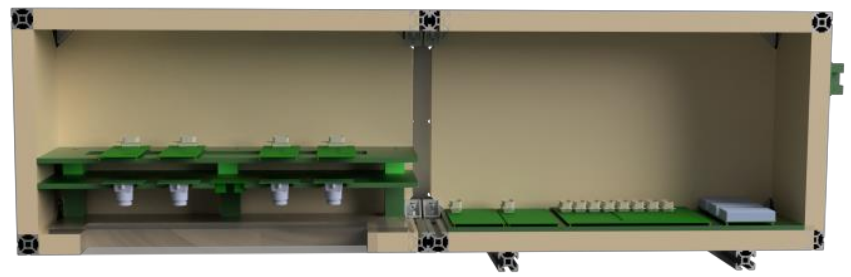

Fig. 2. Mechanical Design a real-time overview of the system during flight.

In Fig. 3 you can see a concept overview of all components including the cable connections. The PDU supplies the power to each system via a dedicated connector.

\section{Software Design}

The software is as designed modular which leads to a high interchangeability of the different components for example switching between different algorithm types. Furthermore, the software is divided into three main parts listed below and visualized in Fig. 4.

1. Sensors: Handling data acquisition, pre-processing and transmission to OBC. For a consistent programming effort and communication, a framework must be designed.

2. Main processor: Central part with need to design an interface for an easy use of distinct algorithms.

3. On Board Data Handler: Responsible for tracking data and communicating with the ground station. Is not part of the framework, but essential for the experiment as it is responsible for ground communication and data storage.

In general, the design can handle partly system failures.

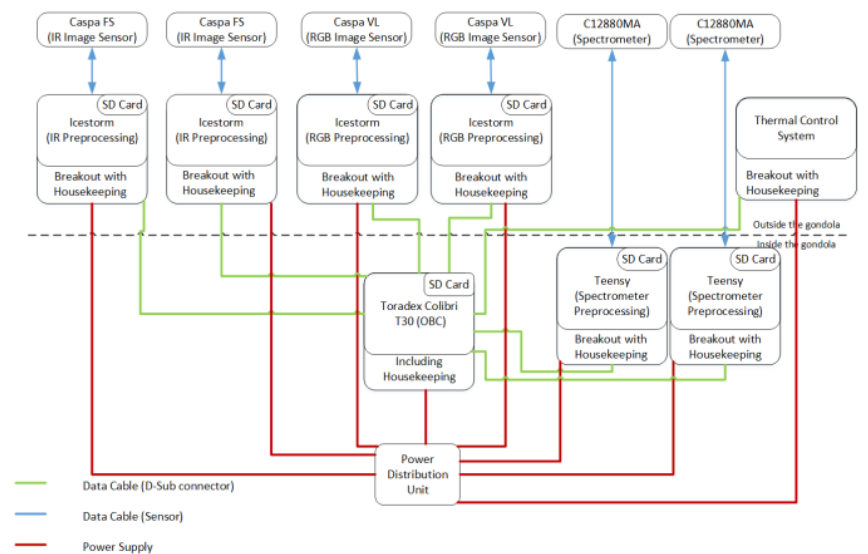

Fig. 3. Electronic Component Overview

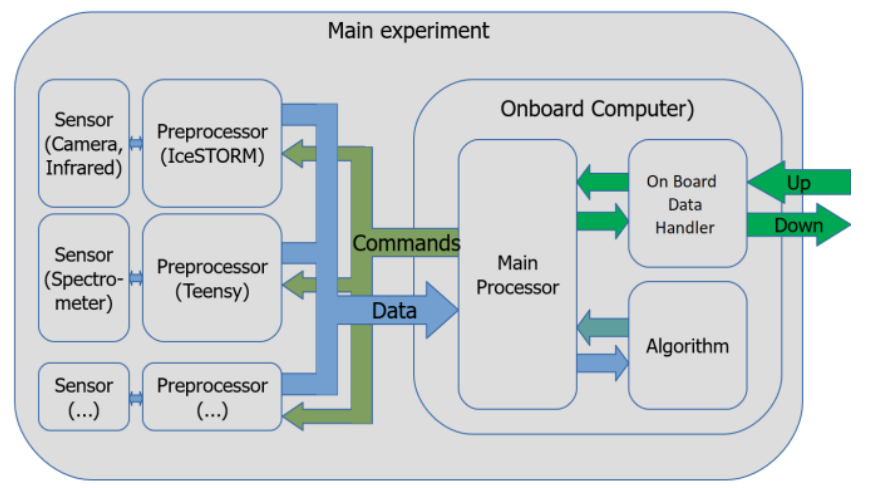

Fig. 4. Software Design 
This means in case of an error on a pre-processor the main processor works with the remaining pre-processors. If the lost pre-processor recovers itself, the data will be used again on the main processor. Furthermore, in case of a main-processor failure each subsystem stores its data locally which means on later postflight analysis the data can be recovered and analysed.

\section{Scientific ALGORITHM}

The central feature of the project is the algorithm. In interplanetary satellite missions one of the most important subjects is the analysis of planet surfaces. Therefor many different sensors are used to get a detailed overview. Nevertheless, the bandwidth for transferring the measurements is limited on a satellite mission. Thus, autonomous evaluation and selection of valuable information onboard of the satellite is required to reduce the resulting data size. Nevertheless, new algorithms to perform this kind of research are rare. Therefore, a new algorithm was developed by the team. The goal was to reduce the amount of data which has to be transmitted and still get detailed knowledge of the surface structure. On earth it would be the differentiation of landscapes, such as water, forest, and acreage and fields. QUEST on BEXUS 27 was the first test flight of this new surface analysis algorithm which can be sketched as followed.

1. The inputs of the algorithm are clearly defined sensor and camera measurements for a given region of the planet surface.

2. These regions are split into small fragments for example the pixels of an image sensor.

3. Then an n-dimensional cluster analysis is performed which groups data points together which have nearly the same coordinates and sensor values. $\mathrm{n}$ is composed of the number of coordinate-axis and the number of distinct sensor measurements for that fragment.

4. The algorithm calculates the mean and standard deviation for each type of sensor value for each cluster in the next step.

5. These values are compared to the reference values which were prepared beforehand, and the most likely category is assigned together with the accuracy to the single points of the cluster.

6. In the last step the clusters are written back in a result image.

As cluster algorithm is used the DBScan algorithm, which works well on high dimensional data sets. See [1] for more information on the algorithm and [2] for more information on the algorithm in multidimensional data sets.

\section{GROUND SUPPORT EQUIPMENT}

\section{A. Ground Support Unit}

For controlling the experiment two tools were developed called the ground support equipment. The Ground Support Unit (GSU) is one part and responsible for the communication between the experiment and the ground. Also, it enables an overview of the system during flight, showing the connection status, allowing to send commands and receive all data, updating the algorithm, see raw data and result images after on board processing.

\section{B. Scientific Support Unit}

The Scientific Support Unit (SSU), shown in Fig. 5, the ground control team can select different clusters in multiple sensor images and classify them with labels. This manually classified data is used to create parameters for the algorithm on board, as well as a database that the algorithm uses for classification, which will be sent to the experiment with the help of the GSU and telecommands. The functionality of the SSU can be described as followed. The SSU allows to overlay images from different sensors of the same image set. A set contains images from multiple sensors that were shot at the same time. The opacity of each image of the set can be controlled individually. The images are shown behind a canvas, which allows to manually draw clusters on top of the images as well as configure the classification of the clusters by drawing with colours for different features. Furthermore, the cluster algorithm can be executed locally on the computer by the SSU to identify clusters which can be assigned to a feature.

\section{FLIGHT ANALYSIS}

During the ascent phase raw images were collected and sent to the ground to update the already existing database, which then was uploaded. The raw images were analysed with the SSU to improve the database continuously. As expected, the predicted features got more and more precise over time. Furthermore, a new integration of a completely new feature was possible during flight. This improved the results, but the final classification at the end of the flight was still improvable which was done during post flight analysis. In general, all components worked as planned. The pre-processors filtered the incoming data to get synchronized and standardized pictures. The communication between the subsystem worked almost fine and all data was stored for later analysis.

\section{A. Image Results}

During the flight, a total of 54 raw RGB image and 54 raw infrared images were downloaded to the ground station. These recorded images are undersaturated and have a round border

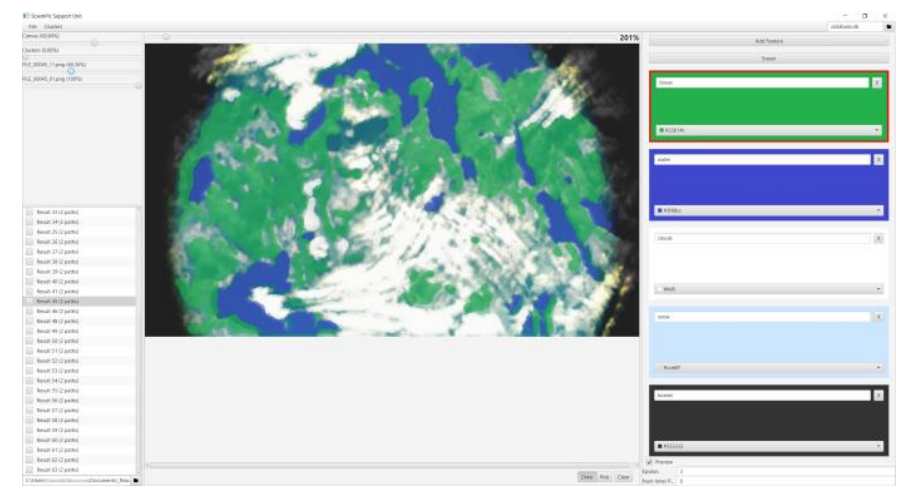

Fig. 5. Scientific Support Unit 
from the filters of the cameras. The RGB cameras also had a slight offset to the infrared cameras, resulting in a translated image. The original images are shown in Fig. 6 and Fig. 7. As expected, the first in flight result images were not satisfying. The by the algorithm yielded clusters were too large and the classification was done incorrectly.

Updates to the parameters of the clustering algorithm as well as updates on classification database improved the quality of the results. This led to a great improvement towards the end of the flight. Still at the end of the flight most clusters were classified incorrectly as a result of the undersaturated images. To increase the quality of the images, a filter and a translation was added during post flight analysis to remedy the effects of the misconfigured cameras. The results of this post processing can be seen in the Fig. 8 and Fig. 9. With the improved images and the improved classification database during the post flight analysis, the algorithm classified the clusters with very few wrong classifications as shown in Fig. 10. The example result classifies the following features:

Water

Forest

Acreage and fields.

Border of the image

Unclassified

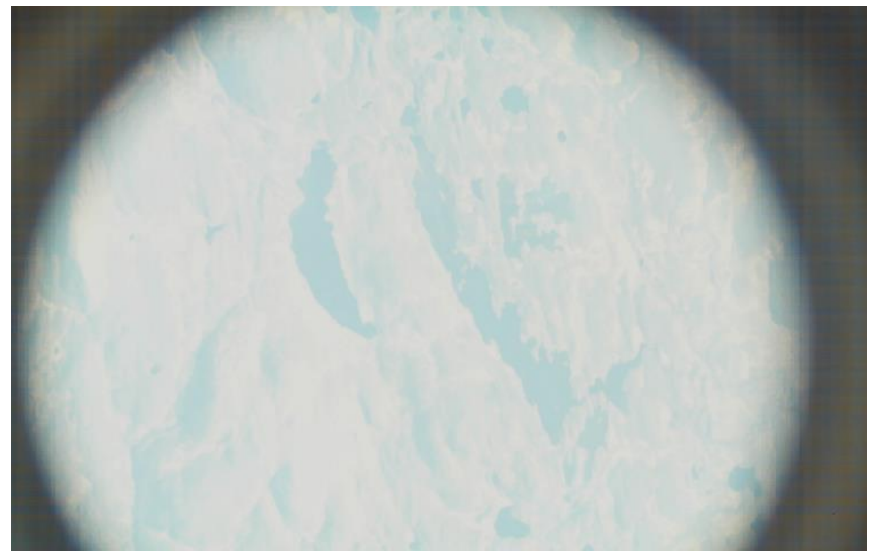

Fig. 6. In flight original RGB image

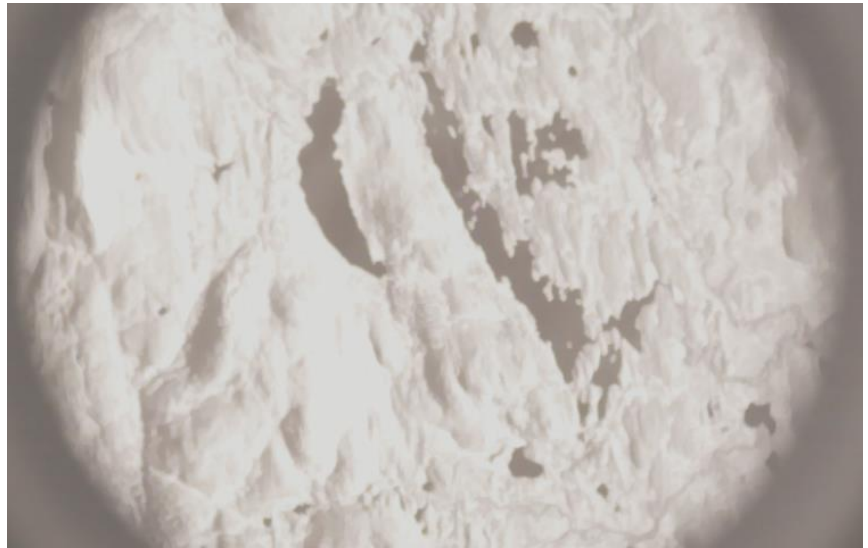

Fig. 7. In flight original infrared image

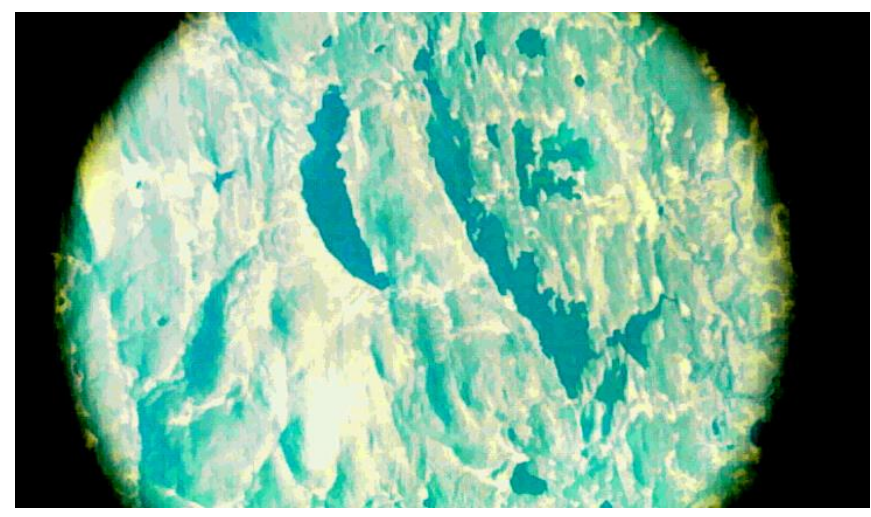

Fig. 8. Post flight optimized RGB image

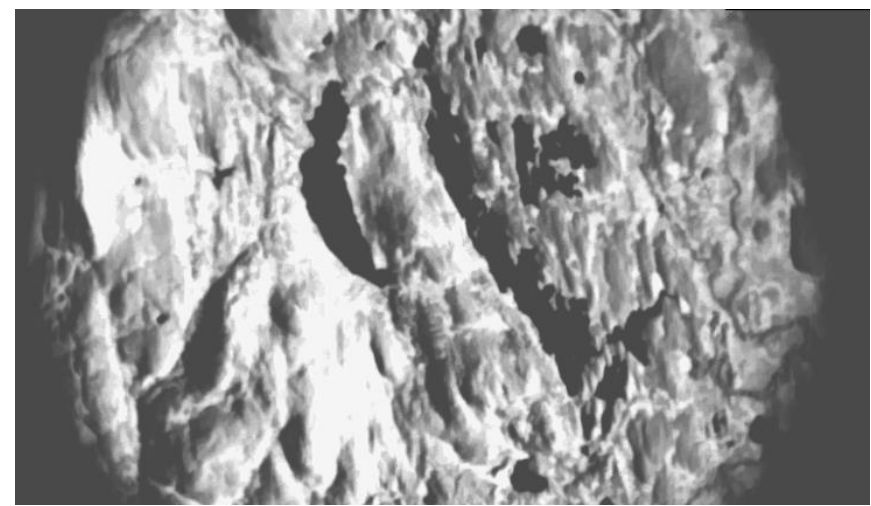

Fig. 9. Post flight optimized IR image

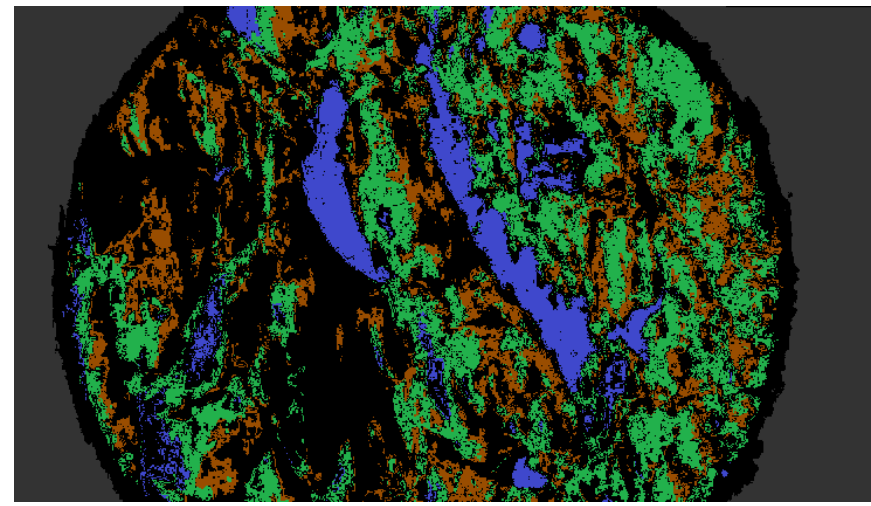

Fig. 10. Result image based on post flight optimized raw images

\section{B. Data Transmission Analysis}

The final data amount is reduced by minimizing the possible values for each pixel of the result image to the number of categorized features. Therefore, the value range for each pixel gets reduced from 256 to the number of features, which are in our case five. This means the needed storage per bit is reduced to only three bits instead of eight bits. Compression the onboard analysis reduces the amount of data even furher. This leads to a reduction of data per result image to 1-20 percentage of the original data, for one sensor. For multiple sensors and higher resolution, the data amount is reduced linear, since instead of all the different source images only one result image must be sent. For four image sensors it is at the maximum 5\% 
of the original data amount. Furthermore, the uplink for an algorithm database is very small. This absolutely fulfils the goal of reducing the amount of data which is produced and must be sent to earth.

\section{LESSONS LEARNED}

\section{A. System testing and verification}

A lot of time was spent testing all systems in private. In the end the time ran short for complete system tests. It was more difficult to solve the found issues, when combining the subsystems to one big system. For example, there were issues with the pre-processors: They work absolutely fine as single system communication as well as image reception and processing. After integrating these subsystems and activating the image sensors, the communication ran unreliable. In the end some pre-processors had issues with handling both. Probably due to manufacturing difficulties. It turned out that the time schedule for tests after combination of subsystems was too short. Especially, the time for solving all issues. Therefore, it is important to save enough time for testing the complete system. Also, the dark rim on the images and the undersaturated images could have been avoided with more extensive tests after full integration.

\section{B. Issue priorities}

Due to trying to solve a compression issue other tests were done more inaccurate. Unfortunate, other issues were detected afterwards, which could have been solved better. Focusing on the compression issue took time, which could have been used to solve problems, which would have revealed more benefits with smaller effort. This means even while solving last errors in the system it should not be forgotten to set the correct priorities. Even if almost all issues are solved.

\section{General educational benefits}

The project was performed by a team of 17 students in parallel to their bachelor studies. As foundation for the project conduced the knowledge gained from university courses. The REXUS/BEXUS program enabled the possibility to gain practical experience while realising a whole real space project. The theoretical content within the university courses found an application during the studies. All the theoretical things about spacecraft system design, modularity, redundancy, single point of failure, verification, testing, etc. lead to a deeper understanding and importance of different fields. Moreover, the students were able to design their own system working in their own fields of interest no matter if it is team management, constructing and planning a hardware design or dive into recent computer science research areas such as data mining and knowledge-based system. Most importantly a lot was learned about working in a team and completing a project including reviews, tests and a launch campaign.

\section{CONCLUSION}

Summarized the project can be described successful in the main objectives. The system was very agile to changes and had no problems with loss of sensors, which appeared during flight and testing, or other failures in different system parts. The modular concept also provided big advantages for solving problems with sensors or pre-processors. Switching to a spare part was possible with very less effort. Also, the experiment itself worked fine. The pre-processing, the system control, and the algorithm worked very fine onboard during flight. Also, the onboard differentiation of landscapes worked, but due to the sensor problems the onboard results were not really satisfying. Fortunately, with extensive post flight analysis the algorithm results were good and the concept as whole is proved to work. After this first concept approval the sensor diversity can be increased, the power usage reduced, and the processing speed enhanced. All in all, the team learned a lot, was able to test the concept successfully and gathered important experiences.

\section{ACKNOWLEDGMENT}

This research project was supported by the REXUS/BEXUS program. The QUEST team is proud to be part of this program, enjoyed all the workshops a lot and is grateful to have flown on the BEXUS 27 mission in October 2018 from the Esrange Space Center. The REXUS/BEXUS program is realized under a bilateral Agency Agreement between the German Aerospace Center (DLR) and the Swedish National Space Agency (SNSA). The Swedish share of the payload has been made available to students from other European countries through the collaboration with the European Space Agency (ESA). Experts from DLR, SSC, ZARM and ESA provide technical support to the student teams throughout the project. EuroLaunch, the cooperation between the Esrange Space Center of SSC and the Mobile Rocket Base (MORABA) of DLR, is responsible for the campaign management and operations of the launch vehicles. The authors also wish to thank the endorsing professor H. Kayal (University of Würzburg) and the companies that provided technical and financial support to the experiment team: Mouser Electronics, Inc., Texas Instruments, Inc., Thorlabs Gmbh, ZARGES Gmbh, Leica Geosystems AG, and $\mu$ duino.

\section{REFERENCES}

[1] M. Ester, H. Kriegel, J. Sander, and X. Xu, "A Density Based Algorithm for Discovering Clusters", Institute for Computer Science, University Munich, Germany, 1996

[2] K. Mumtaz, Dr. K. Duraiswamy, "An Analysis on Density Based Clustering of Multi Dimensional Spatial Data" Indian Journal of Computer Science and Engineering, vol. 1, No. 1, 8-12 